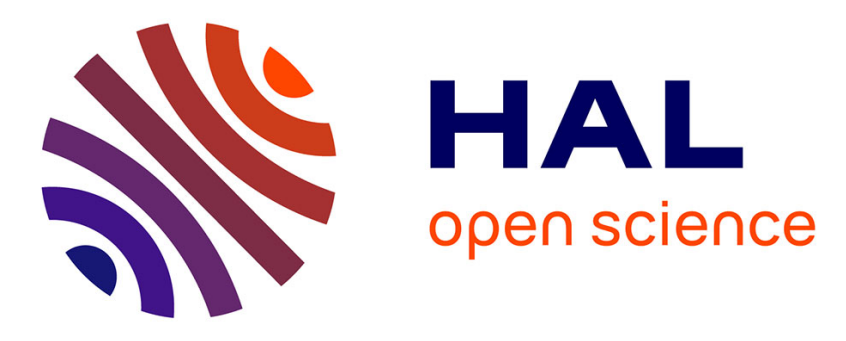

\title{
Implementing a protocol for employing three-dimensional representations in archaeology (PETRA) for the documentation of neolithic funeral architecture in Western France \\ Valentin Grimaud, Serge Cassen
}

\section{To cite this version:}

Valentin Grimaud, Serge Cassen. Implementing a protocol for employing three-dimensional representations in archaeology (PETRA) for the documentation of neolithic funeral architecture in Western France. Digital Applications in Archaeology and Cultural Heritage, 2019, 13, pp.e00096. 10.1016/j.daach.2019.e00096 . hal-02346677

\section{HAL Id: hal-02346677 \\ https://hal.science/hal-02346677}

Submitted on 22 Oct 2021

HAL is a multi-disciplinary open access archive for the deposit and dissemination of scientific research documents, whether they are published or not. The documents may come from teaching and research institutions in France or abroad, or from public or private research centers.
L'archive ouverte pluridisciplinaire HAL, est destinée au dépôt et à la diffusion de documents scientifiques de niveau recherche, publiés ou non, émanant des établissements d'enseignement et de recherche français ou étrangers, des laboratoires publics ou privés.

\section{(c) (1) $\$$}

Distributed under a Creative Commons Attribution - NonCommerciall 4.0 International 


\title{
Implementing a Protocol for Employing Three-dimensional
}

\section{Representations in Archaeology (PETRA) for the Documentation of Neolithic Funeral Architecture in Western France}

\author{
Valentin Grimaud ${ }^{1}$, Serge Cassen \\ $\underline{\text { valentin.grimaud@univ-nantes.fr, serge.cassen@univ-nantes.fr }}$
}

Laboratoire de Recherche en Archéologie et Architectures [Archaeology and architecture research laboratory], Université de Nantes, BP 8122, 44312 Nantes cedex 3, France

\section{Abstract:}

Three-dimensional representation is now commonly used in archaeological studies of ancient structures and objects, and, a fortiori, in studies of the neolithic monumental funeral architecture of Western France. This research relies on mastering several scales of representation: engravings and the materials that are engraved, architecture, topography and the geographical area. However, the datasets produced by techniques such as LiDAR, photogrammetry, laser scans, and even structured-light 3D scans are very dense and difficult to process, and information cannot be extracted from them easily. Further, such documents are used for many purposes, some of which are contradictory: the same digital

1 Corresponding author 
Implementing a Protocol for Three-dimensional Representations in Archaeology (PETRA) for the

Documentation of Neolithic Funeral Architecture in Western France

repository is used for research (for it allows for a maximal amount of information to be obtained), for communication (by producing lightweight representations for real-time display that are visually attractive to site visitors), and for the preservation of images of objects in their current states, along with information about the relations among datasets for future reference (which implies using file formats suitable for long-term retention to ensure that documents can be accessed in the future). This article seeks to present a functional workflow that integrates acquisition, processing, documentation, distribution and guidelines for archiving 3D archaeological data, with a workflow based almost exclusively on free and open-source software. To this end, the authors begin by considering the relationships among three-dimensional datasets, surveying and the documentation of historical sites and artifacts.

Keywords: cultural heritage, 3D documentation, 3D conservation, digitization, real time visualization, free software 


\title{
Implementing a Protocol for Employing Three-dimensional Representations in Archaeology (PETRA) for the Documentation of Neolithic Funeral Architecture in Western France
}

\author{
Abstract: \\ Three-dimensional representation is now commonly used in archaeological studies of \\ ancient structures and objects, and, a fortiori, in studies of the neolithic monumental \\ funeral architecture of Western France. This research relies on mastering several scales of \\ representation: engravings and the materials that are engraved, architecture, topography \\ and the geographical area. However, the datasets produced by techniques such as LiDAR, \\ photogrammetry, laser scans, and even structured-light 3D scans are very dense and \\ difficult to process, and information cannot be extracted from them easily. Further, such \\ documents are used for many purposes, some of which are contradictory: the same digital \\ repository is used for research (for it allows for a maximal amount of information to be \\ obtained), for communication (by producing lightweight representations for real-time \\ display that are visually attractive to site visitors), and for the preservation of images of \\ objects in their current states, along with information about the relations among datasets \\ for future reference (which implies using file formats suitable for long-term retention to \\ ensure that documents can be accessed in the future). This article seeks to present a \\ functional workflow that integrates acquisition, processing, documentation, distribution
}


Implementing a Protocol for Three-dimensional Representations in Archaeology (PETRA) for the Documentation of Neolithic Funeral Architecture in Western France

and guidelines for archiving 3D archaeological data, with a workflow based almost

exclusively on free and open-source software. To this end, the authors begin by

considering the relationships among three-dimensional datasets, surveying and the

documentation of historical sites and artifacts.

Keywords: cultural heritage, 3D documentation, 3D conservation, digitization, real time visualization, free software

\section{The Origins of Architectural Surveying}

The theoretical conceptualization of architectural surveying is generally attributed to the Renaissance artist Raphael (1483-1520). After Pope Leo X had named him the "Prefect" of all the marble and engraved stone of Rome, he laid out the principles of the field (Jones and Penny 1987, p. 205). His Letter to Leo X (see Hart and Hicks 2006) established the basis of a practice that would continue to inform architects and artists for generations to come. Although presenting a detailed history of this period is beyond the scope of the present article, it seems important to note the context in which Raphael wrote to the Pope.

At a time when papal powers were being strengthened, architectural projects were being undertaken throughout Rome, and this produced a great need for construction materials. In particular, Pope Leo X had inherited responsibility for the ongoing construction of the Papal Basilica of St. Peter in the Vatican. This led the period's ancient ruins to be treated as petraia, or quarries, from which an abundance of high-quality stone could be obtained (el-Wakil 2005). However, it was also a time when ancient architecture and art were being rediscovered, and this included several Roman masterpieces. The duties of Raphael's position crystallized the conflict between the need to find materials that could be used in new buildings and the 
drive to conserve the beautiful architecture of antiquity: Leo X had made him responsible for both pursuing ongoing projects and protecting classical marbles.

In his reply to the Pope, Raphael set out the strategy that would be used to preserve the ancient ruins. Paoli (2010, p. 69) outlines his plan as follows, speaking in Raphael's voice: ...my inspection of the ruins has allowed me to understand ancient architecture, and I would like to pass on this knowledge, which is based on my examination of monuments (§§I-V); I must preserve the information that these buildings provide, by drawing reconstructed images of them (§VI); how ancient monuments are selected (§§VII-XII); how these monuments are surveyed and their plans copied (§§XIIIXVII); how monuments can be represented in such a way that they can be measured $(\S \S X V I I I-X X I)$.

More precisely, the working method Raphael established involves three phases (el-Wakil 2005): determining in the field which object to survey; distinguishing ancient ruins from elements added during the Medieval period; drawing the objects of interest in a methodical and meticulous manner, by measuring the proportions of the building directly on the site.

The result of this is a "geometrical description," which entails representing an object or a space using plans, cross sections, and elevations and relating these different views in order to obtain an optimal two-dimensional depiction using the conventions of representation that allow for a mental projection "in 3D." This process calls for a high level of precision at each step, but is indispensable for a true understanding of the volumetric dimensions of the space or object. When a volume is projected on the flat surface of a page, its third dimension, or depth, is necessarily lost. For example — taking only simple geometrical forms into consideration — a circle drawn on a plane could just as easily be the projection of a cylinder, 
Implementing a Protocol for Three-dimensional Representations in Archaeology (PETRA) for the Documentation of Neolithic Funeral Architecture in Western France

a cone or a sphere. Associating an elevation to a plan is what makes the understanding of volumetry possible.

The Letter to Leo X sets out the aim of the procedure of surveying: to create a record by representing ruins that are worthy of interest, in order to produce a trace of the history of the monument and to mark out characteristics in a way that could serve as a point of reference in the future. The representations of architecture thus had to follow specific rules, so that the information could be extracted over time by anyone who wished to do so, for purposes of research, conservation or curating.

\section{Specific Features of the Neolithic Monumental Funeral Architecture of Western}

\section{France}

The neolithic funeral architecture of Western France, and of Europe in general, has a very diverse range of forms. A simplified classification of its types (Boujot 1996) would differentiate between individual sepulchers, which may or may not be accompanied by upright stelae, and collective tombs. However, the fundamental element of all of these constructions is the stone slab, which may be found in a vertical position and in an alignment or in a semicircle, separated from other elements, or within a tomb, serving as the walls of an internal structure. These types can also be combined in any number of configurations, the dimensions of which can vary greatly from one site to another (Fig. 1 and 2). Therefore, if an optimal documentary record is to be obtained, the datasets must take several constraints into account.

The scale of intervention required is also highly variable. A stone slab has various characteristics that can be studied: the traces of extractions (mortises), the extent to which the 
surface has been prepared, and its decorations such as engravings, which may have been made six millennia ago, and may have been protected from the elements, but may also have been exposed to erosive agents such as some kinds of rainfall, wind, and more recently tourism. All of this needs to be documented. These architectural forms can extend over several hundred meters. For example, the well-known Carnac stones (Morbihan), which include the Kermario and Ménec alignments and the Manio quadrilateral, are much larger than the visual field and the horizon; topography is thus an essential parameter for explaining this type of architecture (Cassen 2009). Representing such sites presents several challenges: if the site is drawn in its entirety, it becomes impossible to differentiate the stelae, yet, conversely, representing the details of menhirs prevents the whole of the site from being included in a single document. Tools that provide three-dimensional representations can solve this problem, especially because they can combine several techniques of data acquisition.

Spatial configurations and layouts can vary greatly from site to site and this needs to be taken into account during the process of recording data. The space can be extremely restricted, such as in the main cist of the Mont-Saint-Michel tumulus (Carnac, Morbihan), a funerary space measuring $2 \times 2.4 \times 1 \mathrm{~m}$ at the center of a $125 \times 60 \times 10 \mathrm{~m}$ grave mound. When the mound structure is missing, the pillars and horizontal capstones or "tables" that remain form configurations that are just as complicated to digitize, as is the case for the Pen Hap dolmen (Île-aux-Moines, Morbihan). The dimensions of stelae that are still upright also present a challenge. Finally, some of this architecture, like grave mounds, is very large: the estimated volume of the Mont-Saint-Michel tumulus in Carnac is $30,000 \mathrm{~m}^{3}$. It should be added that the three spatial typologies (convex, concave or planar spaces) can be found independently or all 
Implementing a Protocol for Three-dimensional Representations in Archaeology (PETRA) for the

Documentation of Neolithic Funeral Architecture in Western France

together, depending on the site. This means that data acquisition strategies have to be adjusted to each case.

It should be noted that, unlike most architecture from "historical" periods, these materials were shaped and put together in a way that makes it very difficult to extrapolate missing information. Stelae come from rocky outcrops and although some of their surfaces may have been smoothed out, they do not have the same attributes as dimension stone, which has been carefully cut and worked on in order to be used in rectilinear buildings. Signs of premegalithic, post-megalithic and anthropic stone-working methods can be juxtaposed on the same slab, in such a way that it is very difficult to make extrapolations about what is not visible.

\section{Technical Contributions of Digital Datasets}

\subsection{Documentation using $3 D$ models}

In recent years, as a result of the increased computing power of modern processors, there have been many advances in the tools that produce optical datasets. The precision and quantity of details that can be recorded in a short time are no longer comparable to what can be obtained manually. The choice of what was to be treated used to be made on-site, and therefore observation was a crucial factor. Today, the process has been reversed because large quantities of information are now obtained in the field and the choice of what will and will not be documented is made later. As a result, more time and effort are spent in looking at the digital models of objects than at the real objects themselves. It is also possible to obtain datasets on several scales and at several resolutions and to consolidate these within the same system of spatial coordinates (Pinçon et al. 2010). Concretely, 3D representation allows for 
both the establishment of a digital repository fueled by direct field observation and the development of several approaches to the same object — using "as recorded," "as built," and "reconstruction" models (Fuchs 2007). The integration of all such 3D models into a single virtual environment opens up a new area of research, one that is based not only on covisibility but also on the perception and discovery of monuments when moving from place to place.

Three-dimensional data acquisition and modeling tools have led to a significant diversification of the types of documents that are produced. The 3D medium allows objects and ruins to be explored from many different of points of view, in parallel projection, or in perspective. It is difficult to gauge whether archaeologists have abandoned the geometrical description of architecture or whether it has simply been lost in the vast numbers of images that are produced. This impression is reinforced by the fact that many scientific publications and presentations have come to rely on perspective views rather than on plans, cross sections and elevations. The prevailing impression is that new media have become an end in themselves, and there is no evidence that other types of documents are being produced in order to study objects. Before 3D representation became vastly used in archaeology, geometrical description were commonly used. Then we can assume that technical knowledge are too different between hand-drawing documentation and computer-generated images. A new set of skills in infographics is required, and vocabularies may introduce some semantic confusion since "3D modelling" and "3D documentation" are used in a same way. The work is stopped when the 3D model is completed, and studies are done almost "on the screen", before the creation of a geometrical description. 
Implementing a Protocol for Three-dimensional Representations in Archaeology (PETRA) for the Documentation of Neolithic Funeral Architecture in Western France

However, this definition needs to be clarified, for interactive visualization does not correspond to the standards of architectural description established by Raphael. Modern3D media allow us to produce images that move, but these are difficult for us to pin down and focus our attention on. As a result, they do not enable us to accumulate knowledge in the traditional sense because they prevent us from making comparisons. This is why we find it important to stress that three-dimensional data acquisition and representation techniques do not cancel out the long tradition of documenting places and things with the sort of geometrical description that has been developed since the Renaissance. Rather than replacing traditions, modern techniques are complementary, for they allow us to open up new avenues of reflection.

\subsection{Understand, Preserve, Communicate}

The multi-scale and spatially coherent form of description should serve the three objectives that were already implicit in Raphael's approach: to understand, to preserve and to communicate. It should also be stressed that 3D acquisition and processing procedures of any kind of object require significant investments of money, time, skills and energy. This is why the justification for choosing three-dimensional documentation is so often based on a threephase argument, even if the objectives can conflict with each other.

3D models permit an object to be understood by establishing its dimensions, shapes, and the spatial relations of its different components. Hypotheses about reconstruction and use can be tested without affecting the archaeological ruins themselves. The process begins with the collection of a maximal amount of information and involves treating massive quantities of data. 
The results of this approach should contribute to the establishment of adequate measures for the preservation of the ruins. However, the undeniable advantage of the 3D model lies in how it provides a way to save a precise image of an object at the moment it is studied, so that it becomes easy to compare factors such as the action of erosion and other forms of deterioration over the long term. The precondition of this kind of long-term use is that $3 \mathrm{D}$ files be saved in formats that will remain accessible. Unfortunately, softwares used to generate 3D-data have a short life-cycle and doesn't always exist anymore some years after the study. Therefore, the use of open formats are always strongly recommended, and data should not be stored in proprietary formats.

Finally, the same models can be utilized to produce a range of educational and promotional materials that are specially designed to highlight the research conducted on-site. This is not an incidental concern, because it provides a means for creating a continuity between research results and the greater public. Massive volumes of data, however, are not compatible with the kinds of interactive applications that are becoming the norm for distributing content. It is also worth noting that such communication can concern not only wider audiences, but also the interaction among members of a research group and all other professionals whose work touches on historical sites.

The questions raised by the relation between the three objectives cannot be resolved without addressing the ways in which data are treated. Current practices used in related fields can provide insight into possible new approaches and methods (Grimaud et al. 2016).

In what follows, we shall use the neolithic monumental architecture of Western Europe as a concrete example for studying how such a strategy has been implemented. We have chosen this example even though our work to develop an optimized approach for dealing with the 
Implementing a Protocol for Three-dimensional Representations in Archaeology (PETRA) for the

Documentation of Neolithic Funeral Architecture in Western France

constantly-expanding size of files used for interactive media is still in progress. While it is

clear that interactive applications will become much more widespread in the future, we have

opted to economize on performance rather than involving ourselves in a frantic race to

acquire ever-more-powerful equipment; future users of our applications will not necessarily

have devices with specifications that would allow them to run resource-demanding content.

In other words, we hope that the new features of interactive software will also enable ever-

more-complex graphics to be viewed on the widest range of devices and not simply that they

will make it easier to upload bigger files that hold more data for the same scale of

representation. Increased performance should lead to the sharing of more information, such as contextual information about how a stone slab relates to the site and how the site relates to the landscape. This strategy aims to enrich the ways in which 3D representations are shared and used, while rendering them more accessible by making them available to users with midrange, consumer-grade hardware. Our efforts to address all of these issues coherently has led us to create a protocol for employing three-dimensional representations in archaeology (PETRA).

\section{A Protocol for Employing Three-dimensional Representations in Archaeology}

PETRA is a work process that has the characteristics of a data management plan (Cartier et al. 2015; HAL Descartes 2018) and addresses the three challenges encountered in setting up 3D models - to understand, to preserve and to communicate — in a single workflow (Fig. $3)$. 


\subsection{Spatial granularity defined}

The success of the 3D documentation of archaeological ruins depends on precisely specifying a spatial granularity that is consistent with relevant scientific and technical objectives. In the context of the engraved funeral architecture of the Armorican Neolithic, it is essential to master all the scales of representation, from the base component - the stone slab with engravings that can be measured in the sub-millimeters — to geographical areas that can extend over several hundred square kilometers. To this end, we have identified six entities of scale or levels of representation:

1. the Geographical area, in $5 \mathrm{~km}$ tiles,

2. the Topography, in $1 \mathrm{~km}$ tiles,

3. the Surroundings, in multiple $100 \mathrm{~m}$ tiles,

4. the Tomb structure,

5. the Internal structures,

6. the Slab of stone.

This strategy is an optimization of the 3D scene, and not an application of the one called "level of detail" (LOD). There is indeed no substitution of 3D models according to the degree of visibility of the 3D objects. Only one model is generated according to the level of representation; meshes and textures are prepared to be visually continuous. This allow to concentrate polygons on the most interesting part of the model and to create huge scale environment with an efficient frame-rate.

In our work, information about the first two of these entities comes directly from the Institut national de l'information géographique et forestière (IGN) [France's National Institute of Geographic and Forest Information] geographic information system (GIS) dataset 
Implementing a Protocol for Three-dimensional Representations in Archaeology (PETRA) for the Documentation of Neolithic Funeral Architecture in Western France

and corresponds, respectively, to the following fields of the IGN database: "RGE_ALTI_DST

[Large Scale Reference_Altitude_Distance]" (5 km tiles at a resolution of $5 \mathrm{~m}$ ) and the “RGE_ALTI_MNT [Large Scale Reference_Altitude_Digital Elevation Model]” (1 km tiles at a resolution of $5 \mathrm{~m}$ ). This means that it is not necessary to subdivide the tiles again in order to construct the virtual world, which facilitates the movement from GIS data to 3D models. Entities 3 to 6 concern the architectural site itself, strictly speaking, with its surroundings, as separate from its architectonic elements. Finally, we note that while there is an overlap between entities 1, 2 and 3, the meshes of entities 3 to 6 fit together perfectly within each other.

The main sources from which these 6 levels of representation have been created are 3D datasets. The technique used depends on and should be adjusted to the level of detail sought or the dimension of the entity being documented (Fig. 4).

\section{2. $3 D$ processing and isolating the different components to be studied}

The six entities can be classified into two groups, according to the way in which data are processed (Fig. 5). The information coming from GIS can be treated in ways that are simpler and faster to implement, while acquisition of 3D data from archaeological sites requires much more onerous levels of processing.

The protocol outlined below can be redistributed without need for proprietary licenses because the workflow is based almost entirely on a group of free and open-source software (FOSS). This point must be underlined since tools are accessible to anyone, without any problem to access licence. Programs used include QGIS (QGIS Development Team 2018), Meshlab (ISTI and CNR 2016), CloudCompare (EDF R\&D 2017), Instant-Field Alignment 
Meshes (Jakob et al. 2015a), Blender (Blender Foundation 2017), ImageJ (NIH and LOCI 2018) with the DStretch plugin (Harman 2005) and Krita (KDE 2018).

The entities that correspond to the landscape are, first and foremost, planar; their dimensions are defined by the geographical coordinates of a conic projection which can vary in relation to the region of the globe where the architecture to be documented is located. In the case of the funeral architecture of the Armorican Neolithic, the Lambert-93 conic projection (EPSG::2154) and the NGF-IGN69 French leveling network for altitudes were used. The mesh is subdivided as required to fit closely to the topography (Fig. 6). Tiles of 5 $\mathrm{km}$ provide a basic plan without relief or subdivisions, with information about the surface being provided only though textures. The $1 \mathrm{~km}$ tile is subdivided into a $100 \mathrm{~m}$ x $100 \mathrm{~m}$ mesh, the geometry of which molds to the topography. The $100 \mathrm{~m}$ grid (or multiples thereof) of the surroundings is subdivided as many times as is necessary in order to provide an optimal representation of the ground, and places are reserved in it for the models of the site that will nest into the grid. This requires additional subdivisions for a better adjustment of the boundaries. However, unlike the first two levels (geographical area and topography), in level 3 (surroundings), textures are created using the same mode as that used for the second group.

The entities of the second group, which correspond to the architectural site itself, are produced using retopology performed on dense meshes (Fig. 7). The structure of this mesh is much less rigid than that of the landscape, so that the best possible fit with the volumetry of the entity can be obtained. Models that represent tomb structures and slabs come from a strictly identical workflow; only the techniques used to acquire data change. The mesh is composed primarily of quadrangles to facilitate editing (correction, adjustment and completion). However, internal structures are represented by a triangular mesh, which allows 
Implementing a Protocol for Three-dimensional Representations in Archaeology (PETRA) for the Documentation of Neolithic Funeral Architecture in Western France

for a more orderly geometrical network, but is somewhat more difficult to correct manually. While developing and implementing this protocol, we came to see how to allocate different types of mesh in order to achieve optimal granularity relative to processing time.

Special attention needs to be given to the origin point of these different meshes (the red point in Fig. 5). Since we are working with geo-located data, coordinates are really big and graphic cards are not enough powerful to compute in real time millions of points or triangles. The solution consists to translate models towards the origin of the scene, and to keep the memory of the translation in a paradata file.

- Geographical area (1): The origin of this mesh is located at the $(0,0,0)$ coordinates of the scene center and is at the center of the geometrical plane that is presented without any relief. Several planes with these characteristics can be combined to set up a virtual world that is larger than a $5 \mathrm{~km}$ tile; in this way, a geographical coordinate is defined as the origin of the virtual world and a translation allows all tiles to be positioned correctly.

- Topography (2): the origin of the $1 \mathrm{~km}$ tiles, which provide a way to suggest the topography of the land, has exactly the same characteristics as the tiles of entity 1 . It should nevertheless be noted that if there is any representation of relief, the origin of the model always corresponds to the 0 of the chosen projection system. This means that the translation into $\mathrm{x}$ and $\mathrm{y}$ coordinates is all that is needed to tile the grid of the virtual world.

- Surroundings (3): the scope of this mesh is defined by entity 2, but translating it into $\mathrm{X}, \mathrm{Y}$, and $\mathrm{Z}$ allows the geometry proximal to the $(0,0,0)$ coordinates of the scene 
center to be preserved. This entity enables the system of coordinates of the site (SCS) to be defined.

- Tomb structure (4), internal structures (5) and the stone slab (6): all of these data are consolidated in the SCS.

In this case, three translations are applied: one for the geographical area, one for the topography, and one for the site which includes surroundings, tomb structures, internal structures and stone slab.

Whichever entity is concerned, certain operations can be grouped together and processed as a block (the boxes outlined in red in Figs. 6 and 7). This includes point cloud processing, surface reconstruction and documentation, mesh processing and texture processing.

\subsubsection{Point cloud processing}

Raster images that correspond to digital elevation models (DEMs) or are acquired through LiDAR and laser scanning initially produce point clouds that need to be processed (Fig. 8).

LiDAR and IGN data are already geo-referenced, but this is not the default for laser scan point clouds, which require external processing. When a point cloud has no geo-referencing, different acquisition stations are first consolidated into an arbitrary coordinate system and then consolidated in the SCS. Translation and rotation are preserved for later reference in a paradata file to ensure that the operation can be reproduced.

\subsubsection{Reconstruction and documentation of surfaces}

The procedure that allows surfaces to be documented makes use of the images compilées sous éclairages obliques (ICEO, or "images compiled under oblique lighting") method. Related to photogrammetry, the results of this method can be projected automatically on a 3D 
Implementing a Protocol for Three-dimensional Representations in Archaeology (PETRA) for the Documentation of Neolithic Funeral Architecture in Western France

model to produce accurate $2 \mathrm{D}$ documentation, without needing to adjust the image by stretching it (Fig. 9).

We will not provide a definition of photogrammetry here, since it has become a common technique for 3D data acquisition.

ICEO is very effective in detecting patterns in relief (Cassen and Vaquero Lastres 2003, Cassen et al. 2014). It involves taking a greater or lesser number of photographs from exactly the same point of view, in darkness but with variable luminous intensity, with homogeneous lighting of the scene used only in the first photo. Integrating this ICEO image into the group of photographs used in photogrammetry makes it possible to invert the initial photo with any layer of information produced and thus allow it to be projected onto a 3D model (Domingo et al. 2013). For this, it is enough simply to keep track of the orientation and the dimensions of the image that contains the new information, because its parameters need to be exactly identical to the original.

A collection of ICEO images makes a topographical study of the engravings possible in several steps: the detection of contours (the contrast line between the zones that are over- and under-exposed), percussion impacts, places where material has been removed, and the identification of signs and patterns.

\subsubsection{Mesh processing}

Mesh processing provide a way to reduce considerably the size of high-resolution datasets through the use of retopology (Fig. 10). This allows information about the volumetrics of the object being studied to be separated from information about its surface. All data are usually presented in high-resolution models, but research is optimized when some parameters can be explored individually, without having to take all available information into account at once. 
Before moving to that stage, however, we must ensure that the acquired data are correctly consolidated within the SCS defined by entity 3 (the surroundings).

Retopology was first developed in the context of animated 3D modeling, to prevent the distortion of meshes from creating unwanted folds or creases in the moving image. It involves using quadrangle rather than triangle mesh, and placing the sides of the mesh parallel to the expected distortions. To give a concrete example from the computer graphics industry, an initial model is generated without any concern for its structure and then modified until an aesthetically satisfying result is obtained. High-resolution datasets are composed of a large number of triangles that express, simultaneously, information about the volume and the surface of an object; 3D datasets in archaeology are of precisely the same nature. In a second phase, a new mesh, which is created in relation to a new structure - ideally, one suitable for animation — is defined by tracing the first model. Although animation is only rarely the goal in anthropology, this method allows the number of faces or sides of an object to be reduced considerably, usually by $99 \%$ in our applications. This can take a very long time when done manually. In archaeology, there is no need to obtain perfect retopology, and this work can be automated by using Instant Field-Aligned Meshes (Jakob et al. 2015b).

In general, retopology, which produces a quadrangular mesh, is preferable to the decimate modifier, which results in a triangular mesh. These two transformations yield meshes that are much more lightweight, and therefore much simpler to view and share, although only quadrangles are easy to edit. It is very rare for the different components of an architectural structure to be recorded in their entirety with three-dimensional acquisition techniques. For example, the base of a menhir cannot be recorded if it is upright, but it may have been documented before it was restored by being stood back up (Cassen and Grimaud in press, Fig. 
Implementing a Protocol for Three-dimensional Representations in Archaeology (PETRA) for the Documentation of Neolithic Funeral Architecture in Western France

2). Architectural configurations can also change over time. It then becomes essential to have a means of handling different construction elements to plan for future reconstruction efforts. However, the complexity of internal structures (entity 5) is better expressed with triangular rather than quadrangular mesh, because it produces fewer geometrical errors. To avoid such errors, the model must be segmented on a block-by-block basis, something that would take far more time than our study permits.

In contrast, using automated retopology can produce geometrical errors, but the majority of these are corrected automatically by deleting isolated points and verifying that the mesh is a correct copy. Afterwards, the 3D model can be completed if it has missing parts, corrected if there are aberrations, and adjusted so that it can fit into the $\mathrm{n}^{+1}$ and $\mathrm{n}^{-1}$ entities.

The 3D model obtained through these processing methods will have lost all surface detail, including color texture and its microrelief. These will be reintroduced in the form of textures, but, before this, it is indispensable to define the coordinates of textures. The mesh is placed in a 3D space defined by $\mathrm{X}, \mathrm{Y}$ and $\mathrm{Z}$ coordinates. Before applying a texture, it must be unwrapped in 2D space defined by UV coordinates, while being careful not to introduce too much distortion (contraction or expansion of the meshes), which would produce variations in the resolution of the texture on the 3D model. To prevent this, seams can be placed in strategic locations, not only to avoid deformations but also to achieve a greater surface continuity and to avoid fragmenting the texture at places that are important for the research.

\subsubsection{Texture processing}

Mesh processing not only eliminates color and photo-realistic texture, but also erases all microreliefs. These two types of information can be "retrieved" using texture baking (Fig. 11). This involves transferring information from one mesh to another in the form of textures 
(Cignoni et al. 1998), using new UV coordinates defined by the operator (Heckbert 1986). Each aspect of a surface can be separated off so that it can be analyzed more fully and its "high-definition" characteristics can be rebuilt as a material. For documenting the neolithic monumental funeral architecture of Western France, we found the following parameters to be indispensable:

- Diffuse map: this concerns the photo-realistic color texture of the entity,

- Normal map: this is a blue map that allows $\mathrm{X}, \mathrm{Y}$ and $\mathrm{Z}$ variations between two surfaces to be transposed in RGB,

- Ambient occlusion map: this map allows levels of gray to be used to indicate how much light is received by different parts of the model.

The first two of these three maps are particularly important because they can be modified in order to extract new information that is recorded in an atlas that is specific to the surface being studied.

- The diffuse map can be enhanced with DStretch, an extension for the ImageJ program based on decorrelation stretch. Variations in color that cannot be perceived by the human eye are revealed and can become the point of departure for mapping out prior or current phenomena on archaeological surfaces.

- The normal map can be used with the Phong Bump Map filter in Krita to relight the surface virtually. In certain cases, this enables one to go beyond the physical constraints that the ICEO method can run up against, such as surface concavity or the difficulty in obtaining a dark environment.

Ambient occlusion cannot be adjusted, but it is ideally suited for making use of the different layers of the atlas generated by the first two operations. 
Implementing a Protocol for Three-dimensional Representations in Archaeology (PETRA) for the Documentation of Neolithic Funeral Architecture in Western France

\subsection{Gathering information}

\subsubsection{File names}

PETRA allows a very large amount of information to be mapped in the form of geographical diagrams that can be applied to a 3D model. To facilitate documentation, it is helpful to define precisely what can be mapped and the way the data are to be named. The nomenclature we have adopted remains sufficiently flexible for new entries to be added as needed.

The information that can be mapped has been structured by means of a diagram (Fig. 12). To maintain readability, only the part related to slabs is presented here and the tree-structure of the "stone deterioration patterns" has been simplified. Its function is to provide an inventory of all the observations that can be mapped on a surface, and to indicate how this map should be named. The map is applied to a 3D mesh in the form of a texture, and the name is a means of establishing the link between these two items.

Mesh names have been formalized as follows:

- For entities 1 and 2: "dem_[dimension]hm_[coordinateX]-[coodinateY].ply"

- For entities 3 to 6 :

“[archaeologicalSiteNumber]_[entityId]_[mesh Name]_[acquisitionDate].ply” Maps are named after the complete name of the 3D file, to which “..._themeTag]_[imageId].jpg" is appended. The "themeTag" is located in the upper part of each box and is composed of three letters; the "imageId" is located within the box and is composed of two or three letters, which are sometimes accompanied by a numerical value that expresses the setting used when the image was generated. 
The tree structure of maps is set up in reference to several research efforts, including the International Council on Monuments and Sites (ICOMOS) glossary of stone deterioration patterns (Vergès-Belmin 2008) and Sellier's (1991) inventory of the natural processes involved in the weathering of granite, a stone used frequently in Western France.

\subsubsection{File organization}

Documenting a site in 3D produces a very large number of files. Putting folders into a tree structure provides a coherent way of organizing all of these documents, thus expediting the work process and facilitating the resumption of a project, whether by the same user in the medium term or by another user (Fig. 13). The tree structure has several parts and is organized around the different steps of the workflow delineated in the Consortium 3D SHS [a French social sciences and humanities research consortium focusing on 3D] white paper (Vergnieux et al. 2017). Datasets should be stored in a file format that will remain accessible over the long term and is suitable for archiving: these are A0 files. Once the datasets have been processed in a series of steps, a dense 3D model — which may be a point cloud or a mesh - is produced. This would be a version- 0 file in the present case, which corresponds to an intermediate state of processing prior to the applications of mesh and texture treatment that will produce a version 1 of the representation of the site. There can be as many version- 1 files as there are "as recorded," "as built," and proposed "reconstruction" models. A version-2 file concerns models that have been vetted scientifically by various members of the research and the broader scientific communities.

The structure tries to be as flat as possible and uses a file-extension system to organize files logically, which means that one part can include several folders with the same extension. 
Implementing a Protocol for Three-dimensional Representations in Archaeology (PETRA) for the Documentation of Neolithic Funeral Architecture in Western France

- Section 0: Contains general information about the documentation project, such as administrative documentation or images that illustrate the documentation procedures, including, for example, contextual photos or screenshots of 3D processing in progress, and so on.

- Section 1: Contains all source files for the documentation, converted into an archival format. It also includes laser scan point clouds, photogrammetry and ICEO images, and other archival documents that support reconstruction proposals.

- Section 2: Holds all the files that are used in processing the source data located in section 1. The PhotoScan file for photogrammetry, the VXelements file for structuredlight scans, the Faro Scene LT database for laser scans and so on are also stored here.

- Section 3: For data related to point clouds and dense meshes.

- Section 4: Includes data related to processed, segmented, and structured 3D models. By default, the tree structure has only one folder, but it is possible to add as many as needed, depending on the number of hypothetical reconstructions that are formulated during the research process.

- Section 5: Holds the 3D models that have been scientifically approved by a committee external to the research team.

- Section 6: For all files used to establish a geometrical description of the object of research at several scales of representation.

- Section 7: Contains all possible uses of the 3D model, such as interactive applications or information for rendering the model in a concrete form (with 3D printing, for example). 
- Section 8: Holds paradata files, which include the different settings used for processing data as well as reports written during the study and any accompanying illustrations.

Because this file-system is still under development, its components have been made publicly available on a GitLab instance hosted by the digital humanities research infrastructure Huma-Num (Grimaud 2018). This allows the most recent versions files, templates and project documentation to be downloaded directly such that they do not need to be recreated at the beginning of each new documentation project.

\subsubsection{Keeping track of the work with paradata}

To ensure that the documentation of the site can be reproduced, it is essential to keep a record of a certain amount of technical information. A YAML file (Evans et al. 2009) is used for this purpose because it is the most flexible and rapid way to give structure to short informational messages. This file type can be created with any kind of text editor, but it is most useful to use an editor with syntax highlighting such as Notepad++ (Ho 2018). YAML files used for architectural documentation have the following structure, with data being organized into four parts (Appendix 1):

- Part 1 is limited to identifying the archaeological site, without including all the details about its geographical location or the chronological period of interest, which are other kinds of information to be stored in another file.

- Part 2 describes the characteristics of the 3D source files on which V1 models are based. Documents from archival sources are not included here since longer explanations are required to make use of such material. 
Implementing a Protocol for Three-dimensional Representations in Archaeology (PETRA) for the Documentation of Neolithic Funeral Architecture in Western France

- Part 3 provides a record of all the translations that are needed to reproduce the scene in its entirety, because entities 1 and 2 do not share the same origin as entities $3,4,5$, and 6.

- Finally, part 4 is used to keep track of the characteristics of the camera used to generate the geometrical description. In this way, it remains possible for researchers working at some time in the future to recover exactly the same point of view and thus to continue and complete the project.

\subsection{Ensuring that data remain accessible to future users in the long term}

The importance of archiving 3D models produced in scientific research is highlighted by the publication of international guidelines such as the "Charter on the Preservation of Digital Heritage" (UNESCO 2003) and similar initiatives undertaken by various national and supranational bodies (Vergnieux 2010). The data that are acquired and the models produced could be considered as the digital twin of fragile, real-world archaeological ruins. Although digital conservation cannot in any sense replace the actual ruins, it provides a significant record of the state of a monument at a moment in time, one to which future generations should be able to refer. However, given the heterogeneous mixture of sources, workflows and practices, and results, there is a question about what should be archived, and how.

In response to this, the Consortium 3D SHS has developed aLTAG3D, a Long Term Archive Generator of 3D models (Consortium 3D 2017). This program generates archives of 3D models that are compatible with practices recommended by the Centre informatique national de l'enseignement supérieur (CINES), France's national computer center for higher education. 
The program is designed to function in a highly transparent way. In order for the archiving process to work well, two conditions must be met. First, care must be taken to ensure that the files to be archived have been saved in well-documented formats, which will remain accessible in the long term. This concerns both the source data and the resulting 3D model, along with all related paradata (technical information and scientific arguments). Next, in order to avoid ending up with a large mass of information that needs to be dealt with at the end of a project, the archival repository should be constructed as data processing progresses. This is why PETRA specifies how files should be named and organized.

The archival repository contains the source files, the end results, and the documents that provide a record of how the data were processed and transformed. For this reason, it is essential to establish, within the archive itself, the relation of each of these elements to the others, so that anyone who uses it in the future will be able to follow the thought-processes of the researchers. A 3D archive includes many files that interact with one another in numerous ways, along with somewhat less interesting technical information that must be specified for each file. To make this process easier, a user interface based on boxes that are to be linked together is employed to define the structure of the archive. Many types of boxes are available in the interface, and each corresponds to a particular type of information. The connections between these boxes express the relationships among the different datasets included in the archive. This is also what allows a large number of files to be added quickly. The program can also automatically extract most metadata from files, which significantly reduces the amount of information to be entered manually by users. When the project is finished, it is validated to check that all needed information has been entered correctly. The archival 
Implementing a Protocol for Three-dimensional Representations in Archaeology (PETRA) for the Documentation of Neolithic Funeral Architecture in Western France

repository can then be exported: all data are copied into a folder that can be sent to the service responsible for archiving project data.

The archive created in this way conforms with all CINES standards for archival storage. ${ }^{1}$ However, not all repositories created by this process are intended for this kind of storage. It is important to find suitable partners to participate in data conservation, in accordance with the project and the objects studied.

\subsection{Distributing 3D models}

Another challenge, the last we will address here, concerns how the 3D models created through PETRA can be used. At present, there is a fairly large rift between the tools used by the computer graphics designers who create 3D archaeological models and the tools that allow archaeologists to handle these models. The impact of this gap is even more glaring because the protocol we have just presented generates a huge quantity of information that can be displayed on the 3D model. Although the texture atlas can be consulted in real-time viewing tools, setting up a scene takes a lot of effort every time; the user interface, as well as the interactions and the materials have to be defined. Further, features that would help archaeologists create documentation are missing or need to be developed more fully if they are to become useful. The situation is made even more difficult by the fact that not all research teams working on cultural heritage have the necessary programming skills to meet their specific needs.

To address this, we have used OpenSpace3D (I-maginer 2018), a real-time 3D FOSS rendering engine based on OGRE (OGRE Team 2018). OpenSpace3D is a specialized

1 As the CINES website (2014) notes, "Mandated by its supervisory ministry, the CINES is since 2004 the main actor in the preservation of data and digital documents produced by the French community of Higher Education and Research. It offers digital archiving solutions for medium and long term preservation, solutions which are shared and customizable." 
program for creating applications that make connections between the real world and virtual environments, and its users do not have to write a single line of code. Interactions are set up by using PlugITs, which are blocks of functions that are easy to configure and can be linked together. Once they have been configured, the scene can be exported as an executable for Windows, Mac, Linux or Raspberry Pi, as well as for Android and iOS (some features are not available on all platforms). With a few clicks, it is possible to set up a viewer based on a predefined model that will satisfy the main needs of a professional working with cultural heritage sites. In OpenSpace3D, the entire scene can easily be configured, and it is possible to navigate around objects or visit an architectural site from a first-person viewpoint, to make cross sections, to adjust the virtual light and explore surface detail, to examine the way the sun shines on the site at any time of the year, to export images, and to make annotations on the $3 \mathrm{D}$ model. In this way, it becomes possible to establish nearly all $2 \mathrm{D}$ archaeological documentation within OpenSpace3D.

It also has the advantage of allowing a wide variety of peripherals (virtual reality headsets, Leap Motion controllers, etc.) to be connected to the 3D scene, such that the bridge between the world of research and the contemplative world of museums becomes very easy to cross. OpenSpace3D has great interest both for scientists and for curators and other members of the arts outreach communities, because it proposes forms of interaction that are already configured, but can also be modified in any way one likes.

\section{Application}

PETRA is developed and applied in recent years for our research. The most recent application published can be read (Cassen \& Grimaud 2018; Cassen et al. 2018). We also 
Implementing a Protocol for Three-dimensional Representations in Archaeology (PETRA) for the Documentation of Neolithic Funeral Architecture in Western France

propose to explore the effectiveness of the method through a short case study: the documentation of the Neolithic tomb of Mané er Groez (Carnac, France), better known as Kercado (Fig. 14).

South Brittany (France) is rich of many archaeological structures from the Neolithic period. The compilation of all the 3D reconstructions then makes possible to understand how everything is configured and articulated in the landscape. Beginning with a $75 \mathrm{~km}$ tile facilitates the reconstruction of the universe. Then, $5 \mathrm{~km}$ (entity 1 - geographical area), and $1 \mathrm{~km}$ tiles (entity 2 - topography) are created specifically for a new monument. This strategy allows the creation of very large environments without allocating a lot of resources to its display. The $75 \mathrm{~km}$ tiles and those of entity 1 have the same properties: they are a simple plane; the tiles of entity 2 and 3 (a $100 \mathrm{~m}$ tile for surroundings) are slightly subdivided to express the topography, but most of the information is transmitted with textures (Fig. 14-A, B, C, D, E). All of this data comes from IGN datasets and a LiDAR acquisition from that specific area. A hole is then created inside entity 3 in order to place the documented architecture. In this case, a retopology of high resolution scans (photogrammetry and lasergrammetry, and sometimes structured light) was created (Fig. 14-F, G, H, I). The whole site is thereby described with around $300 \mathrm{~K}$ faces. Each engraved slab is isolated from the entire structure of the tomb, so they can receive an atlas of the textures produced during the archaeological study of the rock art (Cassen et al. 2018, Fig. 14-J).

\section{Discussion}

The protocol we have just described provides an effective way to document the engraved funeral architecture of the Neolithic period in Western France. As we have seen, this protocol combines all six scales of representation, from the sub-millimeter details of engravings to 
geographical areas extending over many square kilometers. Different data acquisition techniques are employed to attain this result, but the list we have presented is not exhaustive and can be supplemented by other methods and programs. We are thinking, in particular, of multispectral imaging, which allows for more precise descriptions of phenomena that took place or are currently at work on surfaces.

PETRA responds to the three challenges of creating 3D models of cultural heritage sites and objects: to understand, to preserve and to communicate. The procedure consists in producing a $3 \mathrm{D}$ model only once, and then using that model to extract surface information to be researched, along with information that allows the different volumes to be put together, according to the spatial granularity desired for the different levels of representation. This becomes possible by splitting off the different components that can be studied: volumetry and surface, as separate from color and relief. A certain number of observations that go beyond the context of the initial study can then be amassed. The data archiving features of aLTAG3D assure that these 3D datasets will remain accessible and understandable to future researchers and can also be reused and completed in the medium term by future topographical studies. This is why PETRA can be considered a data management plan. The accumulation of observations allows adequate conservation measures to be taken more easily. Finally, 3D models extended with texture atlases can be consulted and used with OpenSpace3D to immerse oneself in a scene that represents an archaeological dig or a possible reconstruction, or simply to return to a site virtually, as well as to produce $2 \mathrm{D}$ scientific documentation. This utility of this software is not limited to satisfying the needs of actors in the field of cultural heritage, but can also help with the specific requirements of museum curators and the arts outreach community. 
Implementing a Protocol for Three-dimensional Representations in Archaeology (PETRA) for the Documentation of Neolithic Funeral Architecture in Western France

Based almost exclusively on a FOSS workflow, PETRA is not a rigid protocol; it can be added onto in the future by incorporating new concepts. Finding ways to include the questions of time and of hypothetical reconstruction proposals are themes for future research, which we have not yet confronted. Processing of 3D data has been fully optimized, but certain points still require too much user expertise or take too much processing time. We plan to explore ways to facilitate the assimilation of the entire protocol by new users.

As of now, PETRA has only been tested on ornamented funeral architecture of the Neolithic period in Western France. However, we believe that PETRA is flexible enough to be used to document sites and objects of any size and from any period of history.

Translated by $\underline{\text { John Holland }}$

\section{Acknowledgments}

The authors would like to thank Consortium 3D SHS for providing a context for a broad reflection, beyond the specific concerns of the particular objects we study, and for financing the development of the aLTAG3D and OpenSpace3D programs.
Abbreviations
aLTAG3D a Long Term Archive Generator of 3D models
CINES Centre informatique national de l'enseignement supérieur
FOSS Free and Open Source Software
ICEO Images Compilées sous Éclairages Obliques (or "images compiled under oblique lighting")
IGN Institut national de l'information géographique et forestière (French institute of cartography)
LiDAR Light Detection And Ranging 
PETRA Protocol Empolying Three-dimensional Representations in Archaeology

SCS System of Coordinates of the Site

SHS Sciences Humaines et Sociales (or "Humanities and Social Science")

\section{Funding sources}

This research did not receive any specific grant from funding agencies in the public, commercial, or not-for-profit sectors.

\section{References}

Blender Foundation, 2017. Blender: Open source 3D creation. https://www.blender.org/ (accessed 10 September 2018).

Boujot, C., 1996. Le mégalithisme dans ses rapports avec le développement des sépultures collectives néolithiques. Apports d'une synthèse à l'échelle de la France. Bulletin de la Société préhistorique française. 93-3, 337-341.

https://doi.org/10.3406/bspf.1996.10176.

Cartier, A., Moysan, M., Reymonet, N., 2015. Réaliser un plan de gestion de données: Guide de rédaction (V1, 09/01/2015).

http://cache.media.education.gouv.fr/file/Comment_Participer/36/0/realiser_un_dmp_ 406360.pdf (accessed 9 September 2018).

Cassen, S., 2009. Exercice de stèle, une archéologie des pierres dressées: réflexion autour des menhirs de Carnac, ed. Errance, Paris.

Cassen, S., Grimaud, V., 2018. Enregistrements, représentations et analyse structurale d'une stèle néolithique gravée dans l'Ouest de la France. Kermaillard à Sarzeau (Morbihan, France). Bollettino del Centro Camuno di Studi Preistorici, 43. 
Implementing a Protocol for Three-dimensional Representations in Archaeology (PETRA) for the Documentation of Neolithic Funeral Architecture in Western France

Cassen, S., Grimaud, V., Paitier, H., 2018. Les monolithes gravés dans la tombe à couloir néolithique du Mané er Groez à Kercado (Carnac, Morbihan), Gallia Préhistoire. 58, pp. $87-138$.

Cassen, S., Lescop, L., Grimaud, V., Robin, G., 2014. Complementarity of acquisition techniques for the documentation of Neolithic engravings: lasergrammetric and photographic recording in Gavrinis passage tomb (Brittany, France). Journal of Archaeological Sciences. 45, 126-140. https://doi.org/10.1016/j.jas.2014.02.019.

Cassen, S., Vaquero Lastres, J., 2003. Construction et déconstruction des surfaces sur les temps. Enregistrement et représentation des stèles gravées: Le Bronzo en Locmariaquer et Vieux Moulin en Plouharnel (Morbihan). Revue archéologique de l'Ouest. 20, 109-125.

Cignoni, P., Rocchini, C., Scopigno, R. (1998). A General Method for Preserving Attribute Values on Simplified Meshes. Proceedings Visualization '98 (Cat. No.98CB36276), https://doi.org/10.1109/VISUAL.1998.745285

Consortium 3D, 2017. aLTAG3D - a Long Term Archive Generator for 3D. http://altag3d.huma-num.fr/ (accessed 10 September 2018).

Domingo, I., Villaverde, V., López-Montalvo, E., Lerma, J. L., \& Cabrelles, M., 2013. Latest developments in rock art recording: towards an integral documentation of Levantine rock art sites combining 2D and 3D recording techniques. Journal of Archaeological Science. 40-4, 1879-1889. https://doi.org/10.1016/j.jas.2012.11.024.

EDF R\&D, 2017. CloudCompare: 3D point cloud and mesh processing software. http://cloudcompare.org/ (accessed 10 September 2018). 
el-Wakil, L, 2005. Antique versus moderne au début du XVIe siècle à Rome: La lettre à Léon X, in: el-Wakil, L., Pallini, S., Umstätter-Mamedova, L. (Eds.), Études transversales: mélanges en l'honneur de Pierre Vaisse. Presses universitaires de Lyon, Lyon, pp. 4758.

Evans, C., Net, I. döt, Ben-Kiki, O., 2009. YAML: A human friendly data serialization standard for all programming languages. http://yaml.org/ (accessed 10 September 2018).

Fuchs, A., 2007. Outils numériques pour le relevé architectural et la restitution archéologique (Doctoral Thesis). Université Henri-Poincaré Nancy-I, Nancy, France. Retrieved from https://tel.archives-ouvertes.fr/tel-00145277.

Grimaud, V., 2018, August 27. PETRA. GitLab. Version Control Repository. https://gitlab.huma-num.fr/vgrimaud/PETRA (accessed 25 September 2018).

Grimaud, V., Cassen, S., Rodríguez-Rellán, C., 2016. De l'usage de la représentation tridimensionnelle pour documenter les architectures funéraires monumentales ornées du Néolithique de l'ouest de la France. Les nouvelles de l'archéologie. 146, 23-27. https://doi.org/10.4000/nda.3828.

HAL Descartes, 2018. Réaliser un plan de gestion de données. https://hal-descartes.archivesouvertes.fr/page/data-management-plan (accessed 9 September 2018).

Harman, J., 2005. DStretch: Rock art digital enhancement. http://www.dstretch.com/ (accessed 10 September 2018).

Hart, V., Hicks, P., 2006. Appendix: The letter to Leo X by Raphael and Baldassare Castiglione (c. 1519), in: Hart, V., Hicks, P., (Eds.), Palladio's Rome: A Translation of 
Implementing a Protocol for Three-dimensional Representations in Archaeology (PETRA) for the

Documentation of Neolithic Funeral Architecture in Western France

Andrea Palladio's Two Guidebooks to Rome. CT: Yale University Press, New Haven, pp.177-192.

Heckbert, P., 1986. Survey of Texture Mapping. IEEE Computer Graphics and Applications, 6, pp.56-67.

Ho, D., 2018. Notepad++, a text editor and source code editor for use with Microsoft

Windows. https://notepad-plus-plus.org/ (accessed 10 September 2018).

I-maginer, 2018. OpenSpace3D: Virtual reality for all creative minds.

http://www.openspace3d.com/ (accessed 10 September 2018).

ISTI, CNR. 2016. MeshLab: The open source system for processing and editing 3D triangular meshes. http://www.meshlab.net/ (accessed 10 September 2018).

Jakob, W., Tarini, M., Panozzo, D., Sorkine-Hornung, O., 2015a. Instant field-aligned meshes. http://igl.ethz.ch/projects/instant-meshes/ (accessed 10 September 2018).

Jakob, W., Tarini, M., Panozzo, D., Sorkine-Hornung, O., 2015b. Instant Field-aligned Meshes. ACM Transactions on Graphics. 34, 6, 189. https://doi.org/10.1145/2816795.2818078

Jones, R., Penny, N., 1987. Raphael, ed. CT: Yale University Press, New Haven.

KDE, 2018. Krita: A free and open source digital painting application. https://krita.org/en/homepage/ (accessed 10 September 2018).

Lecornec, J., 1990. Le Menhir orné de Kermaillard à Sarzeau. Bulletin de la Société polymathique du Morbihan. 116, 156-157.

NIH, LOCI, 2018. ImageJ: Image processing and analysis in Java. https://imagej.nih.gov/ij/index.html (accessed 10 September 2018). 
OGRE Team, 2018. OGRE: Object-oriented graphics rendering engine. https://www.ogre3d.org/ (accessed 10 September 2018).

Paoli, M., 2010. La Lettre à Léon X comme ‘discours de la méthode’ ou la restauration de l'architecture antique au moyen du dessin. Scholion. 6, pp.53-76.

Pinçon, G., Bourdier, C., Fuentes, O., Abgrall, A., 2010. De la manipulation des images 3D. In Situ. 13. https://doi.org/10.4000/insitu.6814.

QGIS Development Team, 2018. QGIS: A free and open source geographic information system. https://www.qgis.org/en/site/ (accessed 10 September 2018).

Remondino, F., El-Hakim, S., 2006. Image-based 3D Modelling: A Review. The Photogrammetric Record. 21 (115), 269-291. https://doi.org/10.1111/j.14779730.2006.00383.x.

Sellier, D., 1991. Analyse morphologique des marques de la météorisation des granités à partir de mégalithes morbihannais. L'exemple de l'alignement de Kerlescan à Carnac. Revue Archéologique de l'Ouest. 8, 83-97. https://doi.org/10.3406/rao.1991.1137.

UNESCO, 2003. Charter on the Preservation of Digital Heritage., in: UNESCO (Eds.) Records of the General Conference, 32nd Session, Paris, 29 September to 17 October 2003: Volume 1, Resolutions. Paris, pp. 74-77. http://unesdoc.unesco.org/images/0013/001331/133171e.pdf (accessed 10 September 2018).

Vergès-Belmin, V., (Ed.) 2008. Illustrated glossary on stone deterioration patterns. ICOMOS, Paris. https://www.icomos.org/publications/monuments_and_sites/15/pdf/Monuments_and_ Sites_15_ISCS_Glossary_Stone.pdf (accessed 11 September 2018). 
Implementing a Protocol for Three-dimensional Representations in Archaeology (PETRA) for the Documentation of Neolithic Funeral Architecture in Western France

Vergnieux, R., 2010. Sauvegarder les données numériques 3D du patrimoine, in: Vergnieux R., Delevoie; C., (Eds.), Virtual Retrospect 2009. 4. Éditions Ausonius, Bordeaux, pp. 181-184.

Vergnieux, R., Bernard, J.-F., Chayani, M., Abergel, V., Benistant, P., Bergerot, L., et al., 2017. Consortium 3D SHS White Paper. https://hal.archives-ouvertes.fr/hal-01683842 (accessed 9 September 2018).

\section{List of Figures}

Figure 1. Examples of Neolithic monumental funeral architecture in Western France. Exterior view (A, after V. Grimaud) of the main tomb (B, CAD after S. Cassen \& V. Grimaud) at Mont-Saint-Michel (Carnac, Morbihan); view of the Kermario alignments (C, after S. Cassen) from the mill of Kermaux (Carnac, Morbihan); parietal art at Gavrinis (D, Larmor-Baden, Morbihan, after Cassen S.); an example of a dolmen with a fine tumular structure (D): the Mané Groh dolmen (Erdeven, Morbihan, CAD after V. Grimaud).

Figure 2. The stela of Kermaillard (Sarzeau, Morbihan): documentation from before it was restored to an upright position (A, see Lecornec 1990), its current position (B), geometrical description of the slab (C, see Cassen and Grimaud in press), and the 3D reconstruction of the stela.

Figure 3. The different components of PETRA.

Figure 4. Application of data acquisition techniques to the six entities.

Figure 5. The characteristics of the mesh of each entity (this example is the tumulus of Dissignac, Saint-Nazaire, Loire-Atlantique).

Figure 6. GIS data processing. 
Figure 7. Data processing for the archaeological site.

Figure 8. Point cloud processing.

Figure 9. Reconstruction and documentation of surfaces.

Figure 10. Mesh processing.

Figure 11. Texture processing.

Figure 12. Defining maps for stone slabs.

Figure 13. The folder tree structure that allows PETRA to be used in an optimal manner.

Figure14. Application of PETRA for the documentation of Mané er Groez (Carnac, France). Location of the site with a $75 \mathrm{~km}$ tile (A). Simulation of the Neolithic coast in the region of Carnac and location of the "geographical area" (entity 1) (B). Integration of "topography" (entity 2) and "geographical area" (C), and possibility to switch between an aerial view and relief information (D). Integration of "surroundings" (entity 3) and "topography" (E), and "architectures" (entity 4, 5 6) (F). Different point of view of the tomb: the cairn and hemicycle $(\mathrm{G})$, the entrance of the tomb $(\mathrm{H})$, the passage with an indication of the structure of the mesh (I) and the burial chamber with rock art displayed on the slabs (J).

Appendix 1. List of technical metadata collected during 3D processing and its documentation in YAML format, with syntax highlighting provided by Notepad++. 
Implementing a Protocol for Three-dimensional Representations in Archaeology (PETRA) for the Documentation of Neolithic Funeral Architecture in Western France

Appendix 1. List of technical metadata collected during 3D processing and its documentation

in YAML format, with syntax highlighting provided by Notepad++.

\begin{tabular}{|c|c|}
\hline French Localization & English Localization \\
\hline nomDuSite: & siteName:_ \\
\hline numéroArchéologique: _ & archaeologicalNumber: _. \\
\hline ... & $\ldots$ \\
\hline DONNEES_SOURCES: & SOURCE_DATA: \\
\hline \# permet de mentionner les fichiers sources, leurs caractéristiques, et les transformations & \# provides a place to keep a record of source files, their characteristics, and the \\
\hline permettant de reconstruire la 3D & transformations required to reproduce the 3D model \\
\hline IGN: & IGN: \\
\hline - baseDeDonnées: \# ex : RGE ALTI & - dataBase: \# e.g.: RGE ALTI \\
\hline source: \# renseigner le nom de la dalle utilisée & source: \# enter the number of the tile used \\
\hline LIDAR: & LiDAR: \\
\hline - nomFichier:_xyz & -fileName:_.xyz \\
\hline nombrePoints: _ points & pointCount:_ points \\
\hline dimensionX:_m & dimensionX:_m \\
\hline dimensionY:_m & dimensionY:_m \\
\hline dimensionZ:_m & dimensionZ:_m \\
\hline lasergrammétrie: & laserScan: \\
\hline - nomFichier:_.ply & - fileName:_.ply \\
\hline nombrePoints: _ points & pointCount:_points \\
\hline dimensionX:_m & dimensionX:_m \\
\hline dimensionY:_m & dimensionY:_m \\
\hline dimensionZ:_m & dimensionZ:_m \\
\hline déplacement->1hm: & movement $>1 \mathrm{hm}:$ \\
\hline translationX:_ m & translationX:_m \\
\hline translationY:_m & translation Y:_ $\mathrm{m}$ \\
\hline translationZ:_ m & translationZ:_ m \\
\hline rotationX: _。 & rotationX: _。 \\
\hline rotationY: _. & rotationY: ${ }_{-}^{\circ}$ \\
\hline rotationZ: ${ }_{-}^{\circ}$ & rotationZ: _ ${ }^{\circ}$ \\
\hline photogrammétrie: & photogrammetry: \\
\hline - nomChantier: _ & - chunkName: _ \\
\hline nomDossierPhoto: & photoDirectoryName:_ \\
\hline nombrePhoto: _clichés & photoCount:_photographs \\
\hline modèleAppareilPhoto: _ & cameraModel: _- \\
\hline longueurFocaleMin:_mm & minFocalLength:_ mm \\
\hline longueurFocaleMax:_mm & maxFocalLength:_ mm \\
\hline ouvertureMin: f/_ & minFStop: f/_ \\
\hline ouvertureMax: f/_ & maxFStop: f/_ \\
\hline isoMin: iso _ & minIso: iso _ \\
\hline isoMax: iso _ & maxIso: iso _ \\
\hline logicielReconstruction: Agisoft PhotoScan, version & processingSoftware: Agisoft PhotoScan, version \\
\hline consolidation: & registration: \\
\hline - [nomCible]: & - [targetName]: \\
\hline coordonnéeX:_m & coordinateX:_m \\
\hline coordonnéeY:_m & coordinateY:_ m \\
\hline coordonnéeZ: _m & coordinateZ:_m \\
\hline lumièreStructurée: & structuredLight: \\
\hline - nomFichier:_.ply & -fileName:_ply \\
\hline nombreFaces:_triangles & facesCount:_triangles \\
\hline consolidation: & registration: \\
\hline translationX:_m & translationX:_m \\
\hline translation $Y:_{-} m$ & translationY:_m \\
\hline translationZ:_m & translationZ:_m \\
\hline rotationX: ${ }_{-}^{\circ}$ & rotationX: _。 \\
\hline rotationY:_。 & rotationY: _。 \\
\hline rotationZ: - ${ }_{-}$ & rotationZ: _。 \\
\hline$\ldots$ & \\
\hline
\end{tabular}




\begin{tabular}{|c|c|}
\hline French Localization & English Localization \\
\hline SCENE_V1: & SCENE_V1: \\
\hline \# permet de documenter les translations pour reconstruire un environnement complet & \# provides a place to log translations so that a complete environment can be replicated \\
\hline entite1_MNT_50hm $\rightarrow$ mondeVirtuel: & entity1_DEM_50hm $\rightarrow$ virtualWorld: \\
\hline translationX: & translationX:_m \\
\hline translationY:_ $\mathrm{m}$ & translation Y: ${ }_{-} \mathrm{m}$ \\
\hline translationZ: _ m & translationZ:_m \\
\hline entite2_MNT_10hm->MNT_50hm: & entity2_DEM_10hm->DEM_50hm: \\
\hline translationX:_m & translationX:_m \\
\hline translation Y: _ m & translation $Y_{2}$ m \\
\hline translationZ: _ m & translationZ:_m \\
\hline entite3/4/5/6->MNT_10hm: & entity3/4/5/6->DEM_10hm: \\
\hline translationX:_m & translationX:_m \\
\hline translationY:_m & translationY:_m \\
\hline translationZ:_m & translationZ:_m \\
\hline & $\ldots$ \\
\hline DESCRIPTION_GEOMETRALE: & GEOMETRICAL_DESCRIPTION: \\
\hline \# permet de documenter la création des descriptions géométrales & \# provides a place to document the creation of geometrical descriptions \\
\hline [nomObjetDocumenté]: & [documentedObjectName]: \\
\hline - [nomDeLaVue]: & - [viewName]: \\
\hline orthographicScale: & orthographicScale: \\
\hline dimensionsPixel: ${ }_{-} \mathrm{x}_{-} \mathrm{px}$ & dimensionsPixel: $\mathrm{x}_{-} \mathrm{px}$ \\
\hline dimensionsCentimètres: $\mathrm{x}_{-} \mathrm{cm}$ & dimensionsCentimeters: $\mathrm{x}_{-} \mathrm{cm}$ \\
\hline résolution: _ppi & resolution: _ ppi \\
\hline échelleReprésentation: 1/_ & representationScale: $11_{-}$ \\
\hline pointDeVue: & viewpoint: \\
\hline positionX:_m & positionX:_m \\
\hline positionY:_m & positionY:_m \\
\hline positionZ:_m & positionZ:_m \\
\hline rotationX: - ${ }_{-}$ & rotationX: _o \\
\hline rotationY: _o & rotationY: _ ${ }_{-}^{\circ}$ \\
\hline rotationZ: - ${ }_{-}^{\circ}$ & rotationZ: - ${ }^{\circ}$ \\
\hline
\end{tabular}



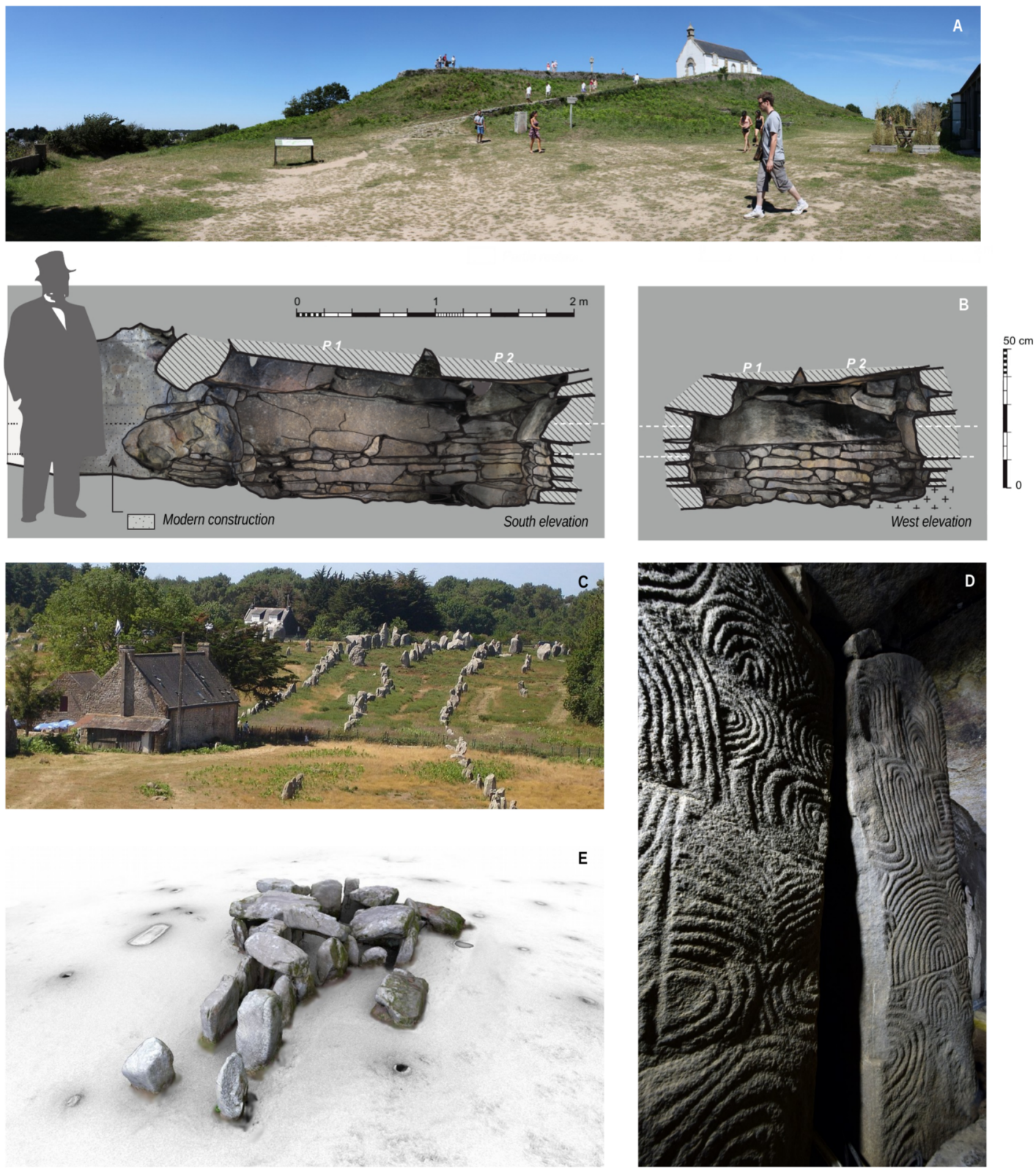

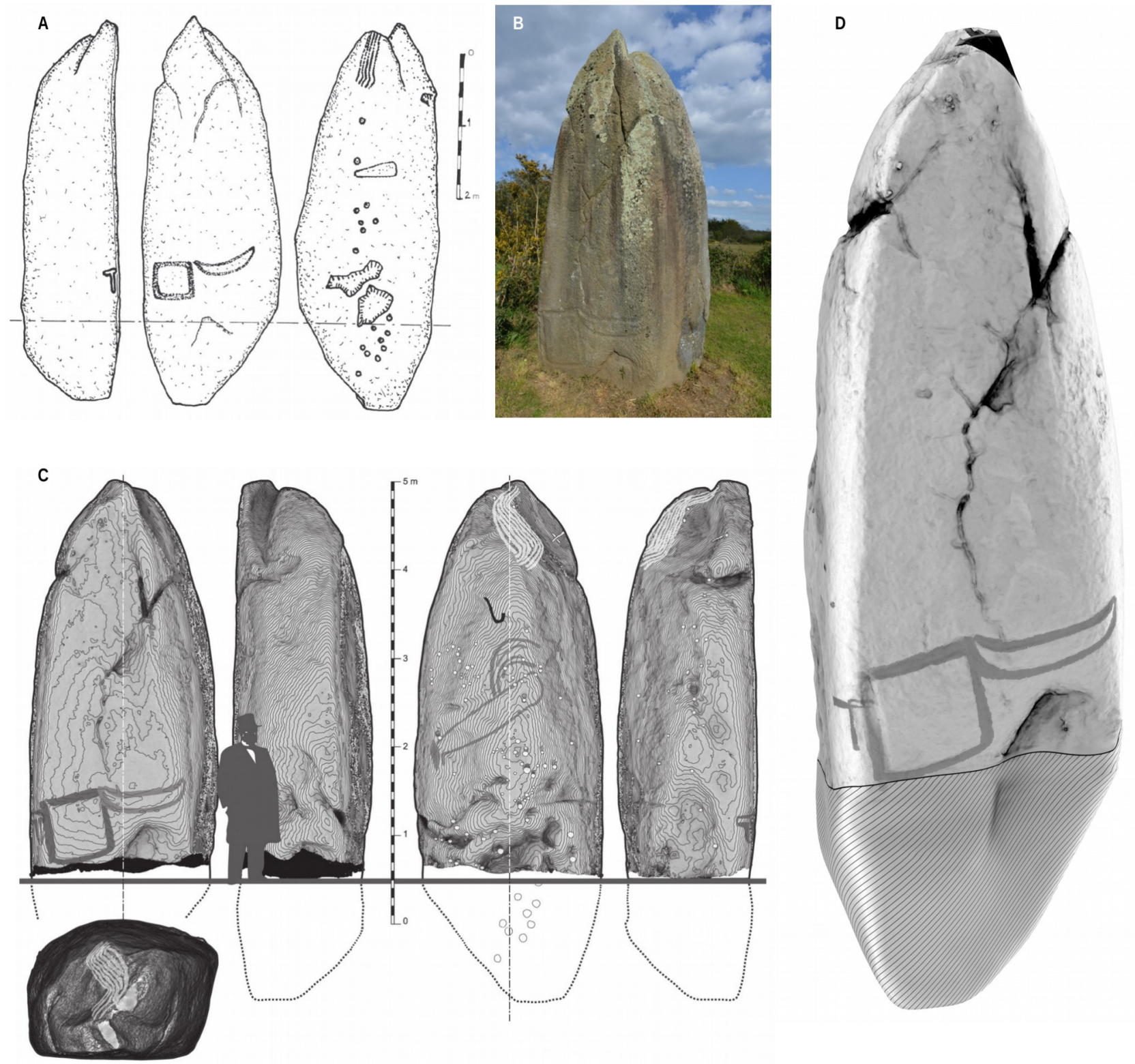


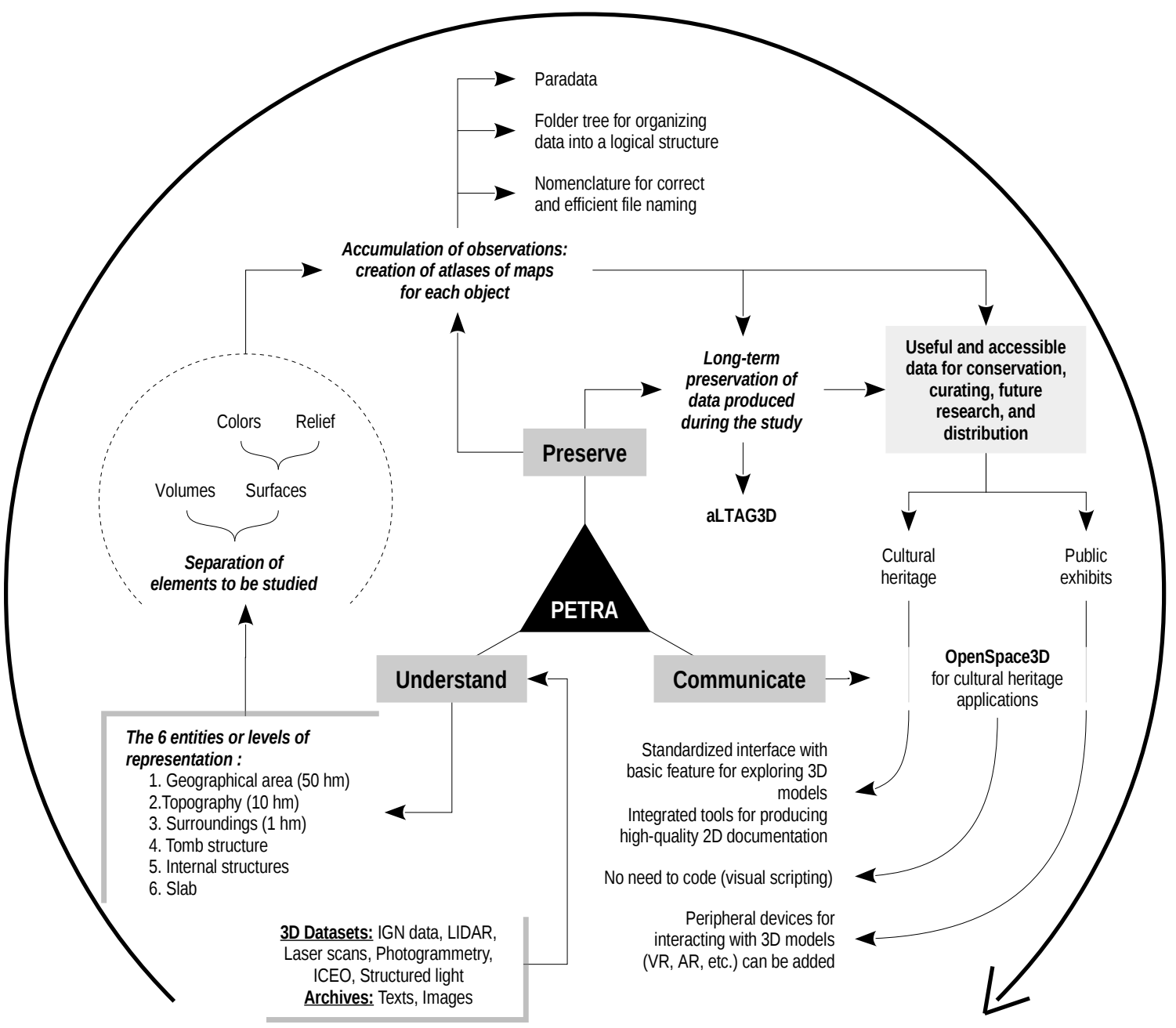




\section{LiDAR}

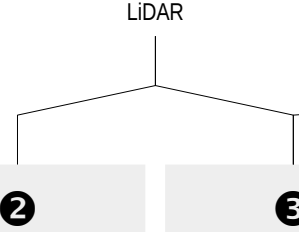

Geographical area (5 km)
Topography $(1 \mathrm{~km})$
Surroundings (100 m)
Photogrammetry

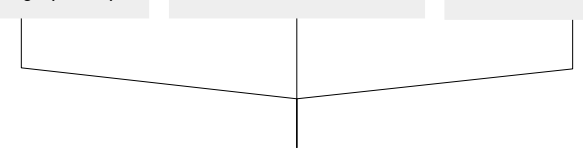

Laser scans

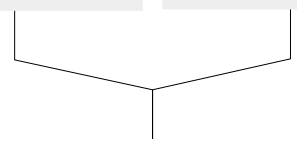

IGN dataset
6

Slab
4

5

Internal structure

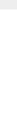

ICEO 
6

Slab (with rock art)

5

Internal structure

4

Tomb structure

3

Surroundings (1 hm)

2

Topography (10 hm)

岩

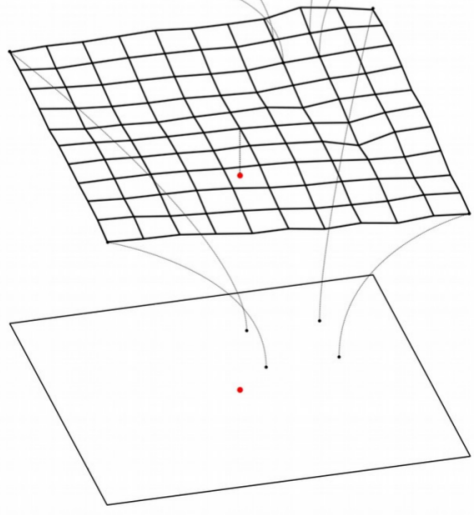

Geographical area (50 hm) 


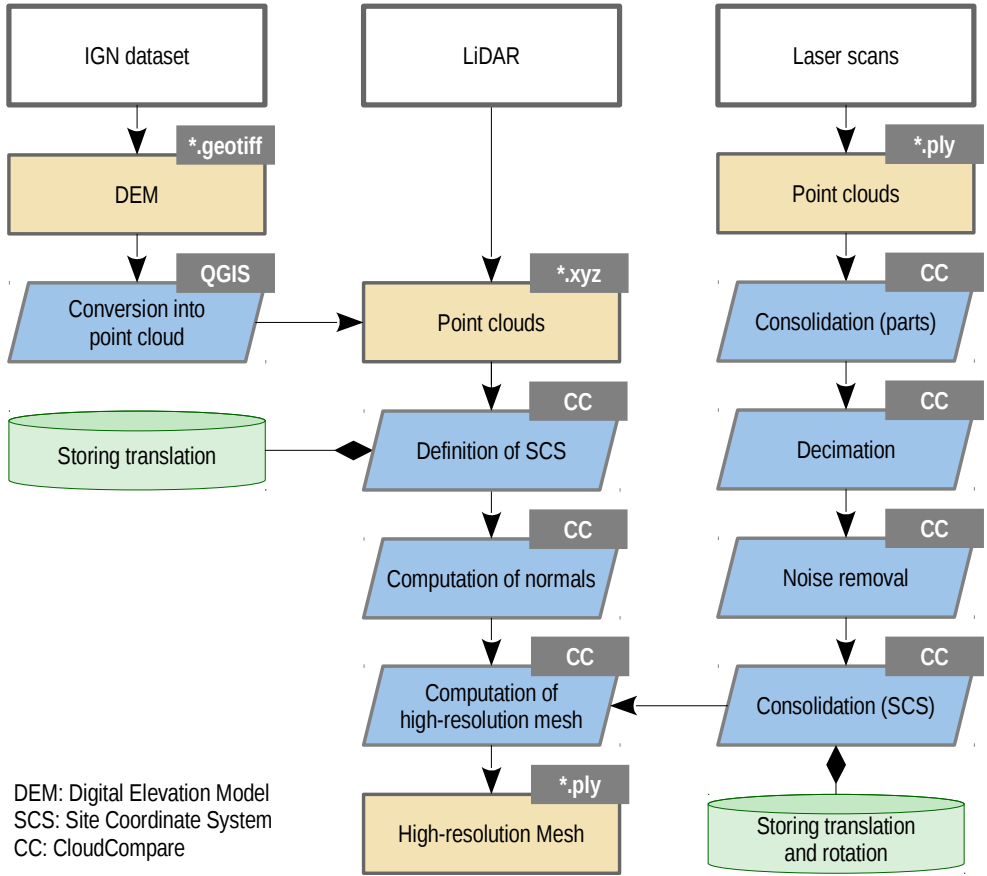




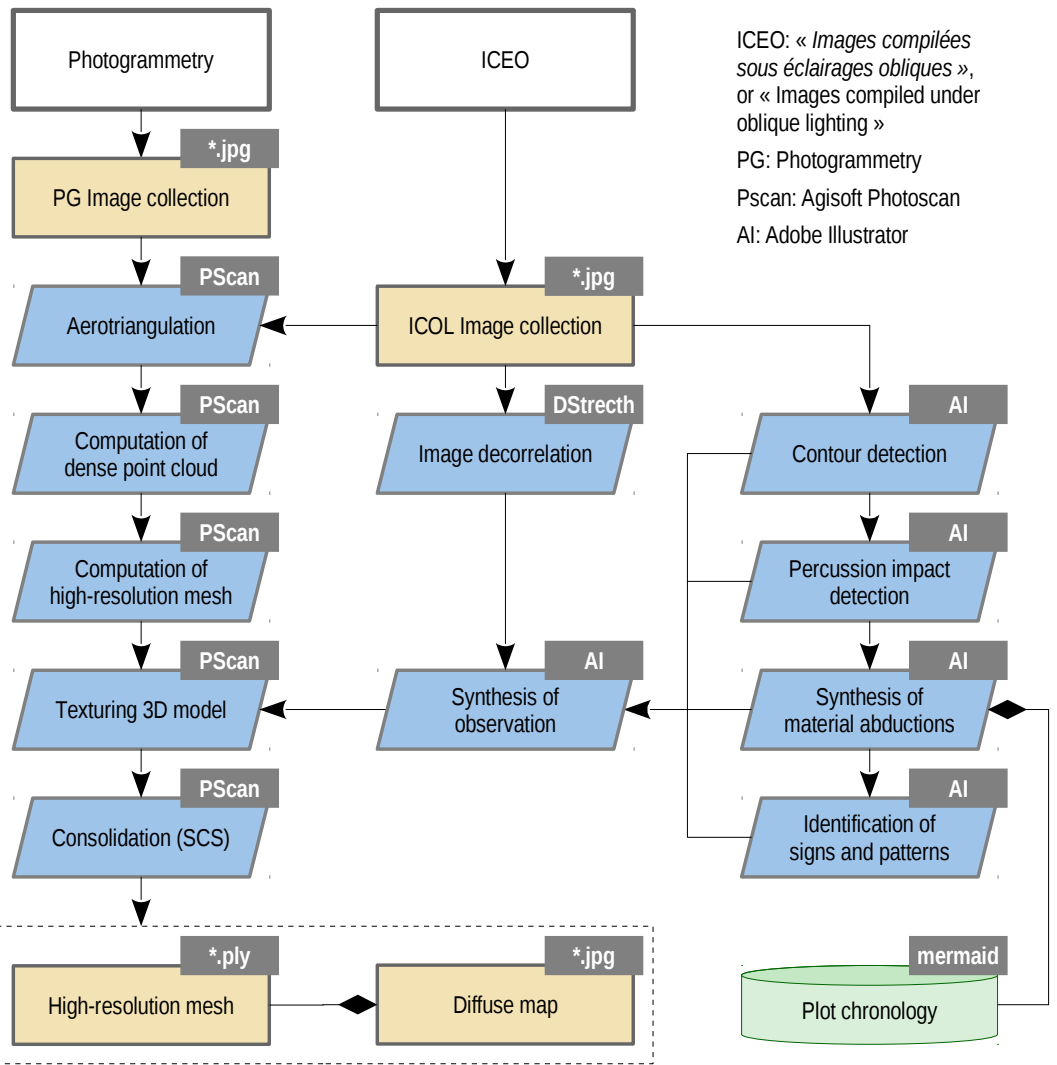




\section{*.ply}

High-definition mesh

\section{Texture}

\section{IFAM}

Retopology

(quadrangles or triangles)

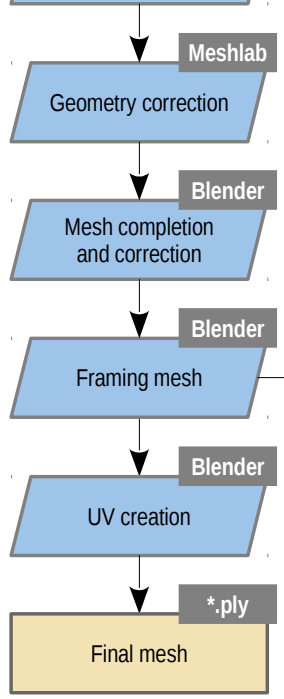

Mesh from the level $n^{-1}$ or $n^{+1}$

Geometry correction = mesh copy and removal of isolated vertices

Mesh completion I correction = deletion of surface irregularities, filling of holes created by missing data 


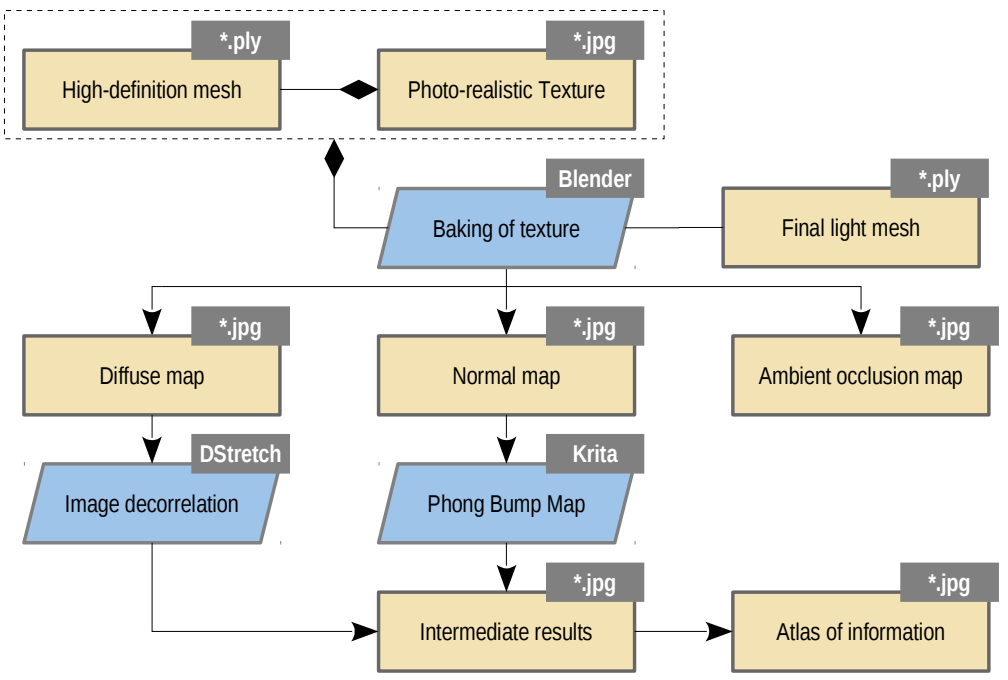




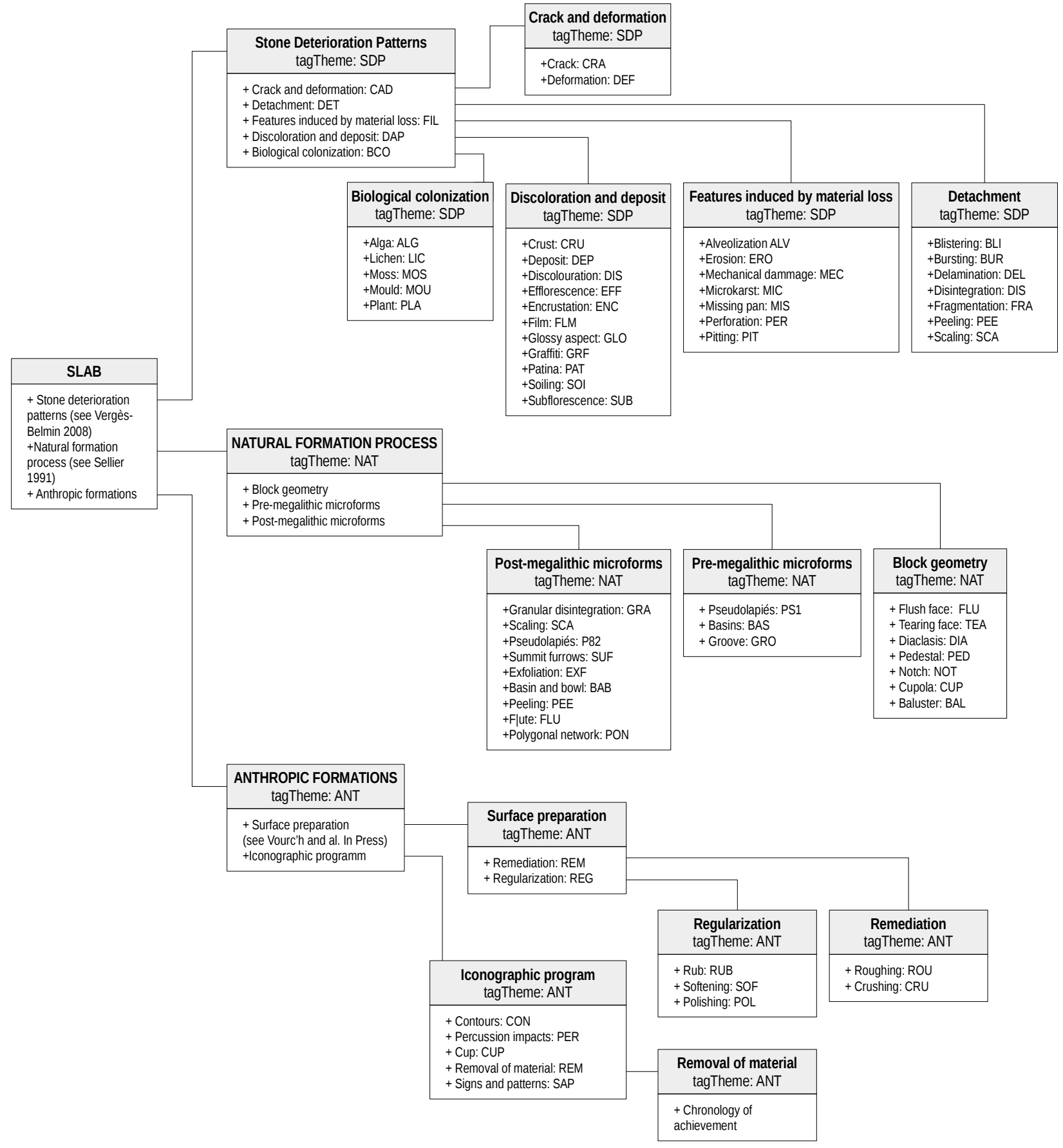


$\llcorner\square$ year_researchProgramName

L $\square 0$ miscellaneous

$\llcorner$ structured in the most logical way

$\square$ 0_administrativeDocuments

$\llcorner$ structured in the most logical way

L1_datasets

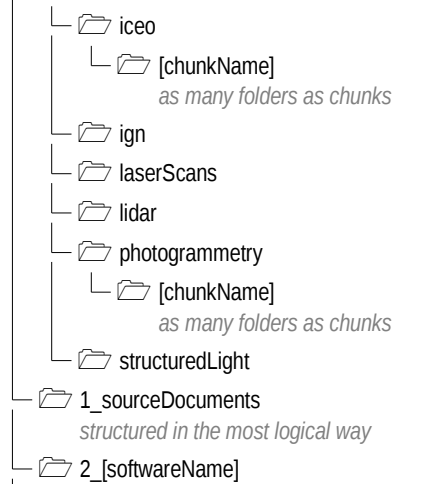

as many folders as software programs or technic used

L 3-1_V0_pointClouds

structured in the most logical way

L 3-2_vo_denseMesh

structured in the most logical way

$\square$ 4-1_V1_documentation

L $\square 30$

$\llcorner\square$ [meshName]

as many folders as meshes

$\llcorner\square$ [slabName]

as many folders as slabs

5_V2_validation
L $\square$ 5_V2_validation

L $\square 30$

$\llcorner\square$ [meshName]

as many folders as meshes

L iceo

$\llcorner\square$ [slabName]

as many folders as slabs

- 6_geometricalDescription

$\llcorner\square$ 1_territory-50hm

$\llcorner\square$ [viewName]

as many folders as views

$\square$ 2_topography-10hm

$\llcorner\square$ [viewName]

as many folders as views

- 3_surroundings

$\llcorner\square$ [viewName]

as many folders as views

L4_tombStructure

$\llcorner$ [viewName]

as many folders as views

L 5 internalStructure

$\llcorner\square$ [viewName]

as many folders as views

\section{6_slab}

$\llcorner\square$ [viewName]

as many folders as view

- 7-1_interactiveApplication as many folders as software used, structured in the most logical way

- 7-2_concreteRenderings as many folders as technique used, structured in the most logical way

- 8_paradata 

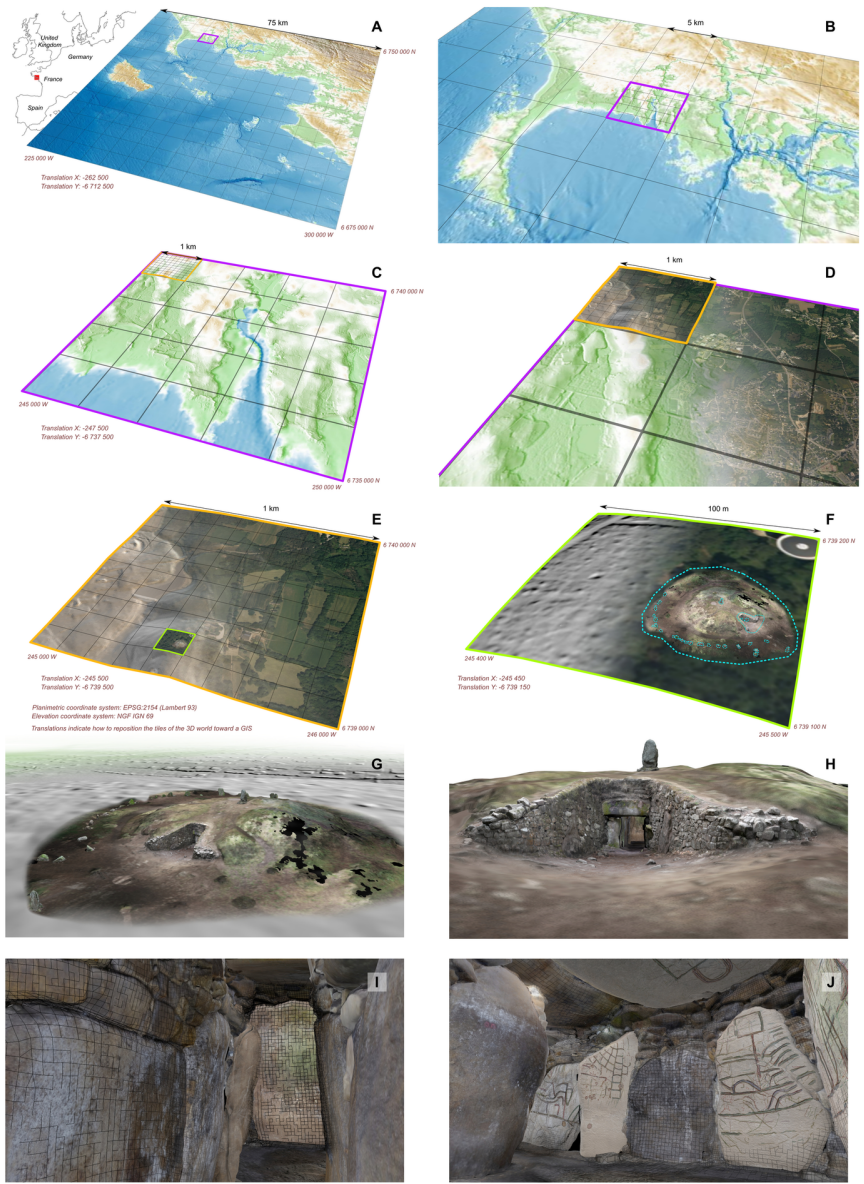